\title{
Hyperoxia reduces STX17 expression and inhibits the autophagic flux in alveolar type II epithelial cells in newborn rats
}

\author{
DAN ZHANG，XINYI ZHAO，DINGNING ZHANG，SIYANG GAO，XINDONG XUE and JIANHUA FU \\ Department of Pediatrics, Shengjing Hospital of China Medical University, Shenyang, Liaoning 110004, P.R. China
}

Received November 28, 2019; Accepted May 5, 2020

DOI: $10.3892 / \mathrm{ijmm} .2020 .4617$

\begin{abstract}
Supplemental oxygen therapy can be life-saving for premature infants. Our previous study revealed a defect in the autophagic flux in the lung tissues of neonatal rats with hyperoxia-induced bronchopulmonary dysplasia (BPD), but the underlying mechanism remains unknown. Moreover, there are few innovative treatments that can completely alter the course of BPD. The present study examined the expression of Syntaxin 17 (STX17), a protein necessary for autophagosome-lysosome binding, in alveolar type II (AT-II) epithelial cells of neonatal rats with BPD. Neonatal Sprague-Dawley rats were randomly exposed to elevated $\mathrm{O}_{2}$ levels [fraction of inspired oxygen $\left(\mathrm{FiO}_{2}\right), 0.8$; model group] or normal room air $\left(\mathrm{FiO}_{2}, 0.21\right.$; control group), and the expression levels of STX17, autophagy-related [Microtubule-associated protein 1A/1B-light chain 3B (LC3B)-II, p62, lysosomal-associated membrane protein 1)] and apoptosis-related (cleaved caspase3) mRNA and proteins were examined in lung tissues. Moreover, the expression levels of the aforementioned proteins were measured in isolated primary AT-II cells cultured in vitro under hyperoxic conditions in the presence or absence of pharmacological modulators of autophagy. Transmission electron microscopy identified that AT-II cell apoptosis and autophagosome aggregation were elevated in the lungs of BPD rats compared with control rats on postnatal day 7. STX17 mRNA and protein expression levels were decreased in lung tissue and isolated AT-II cells as early as postnatal day 3 in BPD rats, while the expression levels of LC3B-II, p62 and cleaved caspase 3 were increased, reaching a peak on postnatal day 7. This early reduction in STX17 expression, followed by increased expression in autophagy- and apoptosis-related proteins, was also observed in isolated AT-II cells exposed to hyperoxia in vitro. However, treatment with the autophagy inducers rapamycin or $\mathrm{LiCl}$ eliminated the hyperoxia-induced
\end{abstract}

Correspondence to: Professor Jianhua Fu, Department of Pediatrics, Shengjing Hospital of China Medical University, 36 Sanhao Street, Shenyang, Liaoning 110004, P.R. China

E-mail: cmufujianhua@163.com

Key words: autophagy, syntaxin 17, bronchopulmonary dysplasia, hyperoxia, neonatal reduction in STX17, partially restored the autophagy flux and increased the survival of AT-II cells exposed to hyperoxia. Collectively, these results indicated that STX17 expression in AT-II cells was reduced in the early stages of BPD in neonatal rats and may be related to the subsequent hyperoxia-induced block in autophagic flux.

\section{Introduction}

Treatment of low birthweight neonates with supplemental oxygen is crucial to their survival, but it is often accompanied by bronchopulmonary dysplasia (BPD), a chronic lung disease predominantly observed in premature infants requiring oxygen and/or ventilation therapy (1). In 2010, the United States National Institute of Child Health and Human Development reported an estimated prevalence of BPD of $\leq 68 \%$ among premature infants with birthweight of 401-1,500 $\mathrm{g}$ and a gestational age of 22-28 weeks (2). Children with BPD experience not only pulmonary dysfunction, but also cardiovascular and nervous system complications that can persist to adulthood (3). Current understanding suggests that the underlying mechanism of BPD in neonates may be related to oxidative stress induced by the shift from the hypoxic environment of the mother to the relatively high-oxygen environment of normal air and/or supplemental oxygen $(1,4)$. The lungs of premature infants have not yet developed a robust protection mechanism and are more vulnerable to damage compared with lungs of infants at full term (4). In addition, oxygen treatment or mechanical ventilation can potentially cause additional lung damage and may interrupt the development of the alveolar and pulmonary vasculature, eventually leading to BPD (4). Therefore, there is an urgent requirement to understand the pathogenesis of BPD in premature infants.

BPD is thought to be caused mainly by intrauterine inflammation, acute lung injury caused by oxidative stress and abnormal differentiation of pulmonary progenitor and stem cells, such as AT-II cells $(5,6)$. However, there are currently no safe and effective treatments for BPD. For example, treatments such as antibiotics for pregnant women or anti-oxidants for neonates have shown no clinical efficacy (7). Glucocorticoids can suppress inflammation and reduce the incidence of BPD, but can also increase the risk of mortality and the incidence of cerebral palsy (1).

Autophagy is a highly conserved cellular recycling process that participates in the degradation of proteins, organelles 
and pathogens (8). The rate of autophagy is influenced by numerous physiological and pathological conditions, including inflammation, cellular stress, apoptosis, transdifferentiation and aging. However, abnormal autophagy activity can also perturbate the balance between cell survival and apoptosis (8). Our previous study showed that AT-II cells from the lungs of neonatal rats with BPD had elevated levels of aggregated autophagosomes and inhibition of the autophagic flux, which contributes to the development of pulmonary tissue dysplasia (9). It was also found that autophagy-inducing drugs may improve lung development in rats with BPD. However, pharmacological modulators of autophagy are not yet in clinical use, and managing their administration to neonates would be more challenging than in adults. Nevertheless, understanding the mechanisms via which BPD inhibits the autophagic flux in pulmonary cells could provide novel approaches for the development of interventions that prevent and treat BPD.

Blockade of the autophagic flux is mainly caused by a failure of autophagosomes and lysosomes to fuse and abnormal degradation of autophagosomes (10). Syntaxin 17 (STX17) is a soluble $\mathrm{N}$-ethylmaleimide-sensitive factor attachment protein receptor (SNARE) protein that promotes the maturation of autophagosomes and binds to another SNARE protein, Vesicle-associated membrane protein 8 (VAMP8), which is essential for the fusion of autophagosomes and lysosomes (10). Failure of this event leads to the accumulation of lysosomes and autophagosomes. Consistent with this, cells deficient in STX17 have been reported to exhibit premature aggregation of autophagosomes $(11,12)$. Based on these previous findings, the aim of the present study was to determine whether STX17 may be abnormally regulated during BPD, leading to the hyperoxia-induced defects in autophagy in the lungs.

\section{Materials and methods}

Animal model of hyperoxia-induced BPD. Sprague-Dawley rats (40 female rats; 8 male rates; weight, 200-240 g; age, 45-65 days) were provided by the Experimental Animal Center of Shengjing Hospital of China Medical University. All newborn rats used for experiments were born to dams at 21-23 days of gestation. Dams were fed a normal diet ad libitum. and housed at a temperature of $22 \pm 2^{\circ} \mathrm{C}$ and a relative humidity of $60-70 \%$, and exposed to light for $12 \mathrm{~h}$ a day. Exposure to hyperoxia was performed as previously described (13). Newborn rats and their mothers were placed in oxygen boxes with either a continuous input of oxygen [fraction of inspired oxygen $\left.\left(\mathrm{FiO}_{2}\right), 0.8\right]$ for the model group or normal air $\left(\mathrm{FiO}_{2}, 0.21\right)$ for the control group. Rats were grouped using random number generation program of SPSS v22.0 software (SPSS, Inc.), with ten rats in each group. The oxygen concentration was monitored continuously and $\mathrm{CO}_{2}$ was absorbed with soda lime to maintain a concentration of $<0.5 \%$. The maternal rats in the model and control groups were exchanged once every $24 \mathrm{~h}$ to avoid differences in feeding ability. Every day, the boxes were opened for $30 \mathrm{~min}$, the padding was replaced and fresh drinking water and food were provided. On postnatal days $1,3,7,14$ and 21, ten rats from each group were selected, anesthetized with pentobarbital sodium $(50 \mathrm{mg} / \mathrm{kg}$; intraperitoneal) and sacrificed. The chest was opened and the lungs were immediately resected. Sections of the left lung were fixed in $4 \%$ paraformaldehyde $>24 \mathrm{~h}$ and $2.5 \%$ glutaraldehyde $>2 \mathrm{~h}$ at room temperature for hematoxylin and eosin staining $(24 \mathrm{~h}$ later; observe under an optical microscope; hematoxylin for $5 \mathrm{~min}$ at room temperature; eosin for $10 \mathrm{sec}$ at room temperature) and immunofluorescence staining, respectively. The right lung was frozen at $-80^{\circ} \mathrm{C}$ before analysis by western blotting and reverse transcription-quantitative PCR (RT-qPCR).

No female rats or newborn rats died in this experiment. However, the BPD newborn rats were not as healthy as the control group newborn rats in terms of weight and breathing status (data not shown). This study has passed and been approved by the Ethical Review of Scientific Research Projects from Shengjing Hospital of China Medical University (approval no. 2019PS321K).

Transmission electron microscope (TEM). Lung tissues were cut into $1-\mathrm{mm}^{3}$ tissue blocks, fixed in $2.5 \%$ glutaral for $>2 \mathrm{~h}$ at room temperature, rinsed with PBS and then fixed in $1 \%$ osmic acid for 2-3 $\mathrm{h}$ at room temperature. Tissues were dehydrated with gradient alcohols $\left(50-90 \%, 4^{\circ} \mathrm{C}\right)$, embedded with acetone embedding medium (TEDIA Company) for 3-4 h at room temperature, sectioned (thickness, 50-60 nm) and double-stained with $3 \%$ uranyl acetate-lead citrate for $30 \mathrm{~min}$ at room temperature. Then, sections were observed at an accelerating voltage of 80-100 kV under JEOL JEM-1200EX TEM (magnification, x20,000; JEOL Ltd.).

Dissociation, purification and culture of primary AT-II cells. The preparation and culture of AT-II cells were performed as described previously (13). On postnatal day 3 and 7, the newborn rats were sacrificed, soaked in $75 \%$ alcohol for $2 \mathrm{sec}$ and the lungs were excised aseptically. Tissue was washed in PBS, cut into $1-\mathrm{mm}^{3}$ blocks, washed again three times with PBS, mixed with $4 \mathrm{ml}$ trypsin and incubated in at $37^{\circ} \mathrm{C}$ water bath for $30 \mathrm{~min}$ with shaking. DMEM (Gibco; Thermo Fisher Scientific, Inc.) containing 10\% FBS (Gibco; Thermo Fisher Scientific, Inc.) was added at $4 \mathrm{ml}$ per sample and the cells were gently homogenized into a single-cell suspension, filtered with a sterile 200-mesh sieve and centrifuged for $5 \mathrm{~min}$ at $200 \mathrm{x} g$ at room temperature. The supernatant was discarded and the cells were resuspended in $5 \mathrm{ml}$ type I collagenase, shaken in a water bath at $37^{\circ} \mathrm{C}$ for $40 \mathrm{~min}$ and centrifuged for $5 \mathrm{~min}$ at $200 \mathrm{x} \mathrm{g}$. The supernatant was discarded and the cells were resuspended in DMEM containing 10\% FBS, $10,000 \mathrm{U} / \mathrm{ml}$ penicillin and $25 \mu \mathrm{g}$ streptomycin. The cell suspension was adjusted to a density of $5.0 \times 10^{5} / \mathrm{ml}$, placed in a $25-\mathrm{cm}^{3}$ culture flask and incubated at $37^{\circ} \mathrm{C}$ in a $5 \% \mathrm{CO}_{2}$ atmosphere to adhere.

Hyperoxia exposure and treatment of AT-II cells. Primary AT-II cells prepared as described in the aforementioned section were incubated at $37^{\circ} \mathrm{C}$ at $\mathrm{FiO}_{2} 0.8$ for $0,6,12,24$ or $48 \mathrm{~h}$ and then harvested for analysis. Rapamycin (RAPA; in DMSO and PBS), LiCl (in PBS), 3-methyladenine (3-MA; in $\mathrm{PBS}$ ) and/or chloroquine (CQ; in PBS) were added at final concentrations of $5 \mu \mathrm{M}, 5 \mathrm{mM}, 5 \mu \mathrm{M}$ and $5 \mu \mathrm{M}$ at $37^{\circ} \mathrm{C}$ for $24 \mathrm{~h}$, respectively. Cells were collected for analysis at the indicated time point of $24 \mathrm{~h}$. All of the aforementioned drugs were purchased from Sigma-Aldrich (Merck KGaA). Preparation of the main reagents is presented in Table I. 
Table I. Preparation of the main reagents.

Reagent name

\section{RAPA}

$\mathrm{LiCl}$

3-MA

CQ
Preparation method

RAPA, rapamycin; 3-MA, 3-methyladenine; CQ, chloroquine.

Immunofluorescence. Lung tissues were fixed in $4 \%$ paraformaldehyde at room temperature for $24 \mathrm{~h}$, dehydrated with gradient alcohol, vitrified with xylene, embedded in paraffin and sectioned (thickness, $5 \mu \mathrm{m}$ ). Paraffin-embedded lung tissue sections were deparaffinized, hydrated, soaked in formaldehyde at room temperature for $30 \mathrm{~min}$, washed three times with PBS, retrieved by microwave with citric acid, washed again with PBS three times and incubated with $10 \%$ goat serum (MXB Biotechnologies, Inc.) at $37^{\circ} \mathrm{C}$ for $30 \mathrm{~min}$ to block antibodies. Then, sections were incubated with primary antibodies (rabbit polyclonal STX17 antibody; cat. no. 17815-1-AP; 1:100; ProteinTech Group, Inc.) overnight at $4^{\circ} \mathrm{C}$. The negative control was incubated with PBS. The following day, sections were washed with PBS, incubated with secondary antibody (cat. no. KIT-9720; 1:5,000; MXB Biotechnologies, Inc.) at $37^{\circ} \mathrm{C}$ for $4 \mathrm{~h}$, washed again with PBS three times and stained with DAPI at $23^{\circ} \mathrm{C}$ for $5 \mathrm{~min}$. Sections were washed with PBS and observed under a fluorescence confocal microscope (magnifications, x200 and x400; MTC-600; Bio-Rad Laboratories, Inc.). Results were evaluated as follows: Red fluorescence indicated STX17 positivity.

Western blotting. Lung tissues were cut into pieces, incubated in lysis buffer at $4^{\circ} \mathrm{C}$ for $1 \mathrm{~h}$ and centrifuged at $4{ }^{\circ} \mathrm{C}$ at $15,000 \times \mathrm{g}$ for $10 \mathrm{~min}$. Protein concentration determination using bicinchoninic acid assay. The supernatant was collected, diluted in Laemmli buffer (Sigma-Aldrich; Merck KGaA) and boiled for $3 \mathrm{~min}$. Proteins (10-15 $\mu \mathrm{l} /$ lane)were separated on $12 \%$ SDS-PAGE for $2 \mathrm{~h}$ at $80 \mathrm{~V}$ at room temperature and then transferred to PVDF membranes for 90 min at $80 \mathrm{~V}$ at $4^{\circ} \mathrm{C}$. The membranes were blocked with $5 \%$ albumin bovine serum at room temperature for $1 \mathrm{~h}$ (Beijing Solarbio Science $\&$ Technology Co., Ltd.) and then incubated overnight at $4^{\circ} \mathrm{C}$ with the following primary antibodies: Rabbit monoclonal Microtubule-associated protein 1A/1B-light chain 3B (LC3B; cat. no. 3868; 1:100; Cell Signaling Technology, Inc.), rabbit monoclonal cleaved caspase3 (cat. no. 9664; 1:1,000; Cell Signaling Technology, Inc.), rabbit monoclonal p62 antibody (cat. no. 5114; 1:1,000; Cell Signaling Technology, Inc.) and mouse monoclonal lysosomal-associated membrane protein 1 (Lamp1; cat. no. sc17768; 1:1,000; Santa Cruz Biotechnology, Inc.). As a negative control, membranes were incubated with Tris- $\mathrm{HCl}$ buffer plus Tween-20 $(0.1 \%)$ overnight at $4^{\circ} \mathrm{C}$. The following day, the membranes were incubated with secondary antibodies (horseradish peroxidase conjugate; anti-mouse IgG; cat. no. 7076; 1:2,000, Cell Signaling Technology, Inc.; Anti-rabbit IgG; cat. no. 7074; 1:2,000; Cell Signaling Technology, Inc.) at $37^{\circ} \mathrm{C}$ for $2 \mathrm{~h}$, and proteins were then detected using ECL substrate (Thermo Fisher Scientific, Inc.). $\beta$-actin (cat. no. 4970; 1:2,000; Cell Signaling Technology, Inc.) was probed as a loading control. Band densities were calculated using Image-pro Plus software6.0 (Media Cybernetics, Inc.).

$R T-q P C R$. RNA was extracted from frozen lung tissues using the TRIzol ${ }^{\circledR}$-acetone (Takara Bio, Inc.) method and then reverse transcribed $\left(37^{\circ} \mathrm{C}\right.$ for $15 \mathrm{~min}$ and $85^{\circ} \mathrm{C}$ for $\left.5 \mathrm{sec}\right)$ using a Takara reverse-transcription kit (cat. no. RR047A; Takara Bio, Inc.) according to the manufacturer's instructions. qPCR was performed using a Takara SYBR-Green kit (cat. no. RR420A; Takara Bio, Inc.) in 20- $\mu 1$ reaction volume with primers designed and synthesized by Takara Bio, Inc. (Table II). The thermocycling conditions were as follows: Initial denaturation at $95^{\circ} \mathrm{C}$ for $10 \mathrm{sec}$, followed by 40 cycles at $55^{\circ} \mathrm{C}$ for $5 \mathrm{sec}$ and $60^{\circ} \mathrm{C}$ for $34 \mathrm{sec}$. The results were automatically analyzed by the PCR analyzer 1.02 (Applied Biosystems; Thermo Fisher Scientific, Inc.) The relative amount of transcripts was calculated using the $2^{-\Delta \Delta \mathrm{Cq}}$ formula, normalized to GAPDH or actin transcript as an internal control (14).

MTT cell cytotoxicity assay. Primary AT-II cells were cultured for $24 \mathrm{~h}$ until 70-80\% confluent, collected and seeded in 96 -well plates at $4 \times 10^{3}$ cells/well ( $n=5$ per condition). Cells were cultured in an incubator under hyperoxic $\left(\mathrm{FiO}_{2}, 0.8\right)$ or normal conditions $\left(\mathrm{FiO}_{2}, 0.21\right)$ at $37^{\circ} \mathrm{C}$ for $24 \mathrm{~h}$ in the presence or absence of autophagy inducers or inhibitors (Table I). At the end of the incubation, $20 \mu \mathrm{l}(5 \mathrm{mg} / \mathrm{ml})$ MTT was added per well and the cells were incubated at $37^{\circ} \mathrm{C}$ for $4 \mathrm{~h}$. The supernatant was removed and $200 \mu \mathrm{l}$ DMSO was added per well to dissolve the purple formazan crystals. The absorbance at $490 \mathrm{~nm}$ was measured using a microplate reader and the data were subsequently analyzed.

Statistical analysis. SPSS v22.0 software (SPSS, Inc.) was used to analyze the data, which are presented as the mean \pm standard deviation. The homogeneity of variance of two samples was analyzed with the F test, and multiple comparisons were 
Table II. Primer sequences.

\begin{tabular}{lll}
\hline Gene & \multicolumn{1}{c}{ Forward primer $\left(5^{\prime}-3^{\prime}\right)$} & Reverse primer $\left(5^{\prime}-3^{\prime}\right)$ \\
\hline LC3B & AGAGCGATACAAGGGTGAGAA & CACTTCAGAGATGGGTGTGG \\
STX17 & CTTGTCATCGTCCGCATTCTG & TCAAGCCTGCGTAGCTTCACC \\
p62 & CTGTGGTGGGAACTCGCTAT & AAGGGGTTGGGAAAGATGAG \\
Lamp1 & TCCAGGCTTTCAGGGTAGAA & ATGAGGACGATGAGGACCAG \\
-actin & CGTGCGTGACATTAAAGAG & TTGCCGATAGTGATGACCT
\end{tabular}

STX17, syntaxin 17; LC3B, Microtubule-associated protein 1A/1B-light chain 3B; Lamp1, Lysosomal-associated membrane protein 1.

performed using one-way ANOVA with Bonferroni's post hoc test. $\mathrm{P}<0.05$ was considered to indicate a statistically significant difference.

\section{Results}

Morphological and ultrastructural changes in the lungs of rats with $B P D$. Light microscopy of lung sections from control rats on postnatal day 1 identified pulmonary alveoli with a large volume and uniform size, as well as moderate alveolar septa (Fig. 1A). Moreover, the ridge-like structures gradually increased, alveoli and capillaries were more abundant and the alveolar septa thinned as the age increased. However, the lungs of BPD rats exhibited notably widened and edematous alveolar septa on day 3, and by day 7 there were fewer but larger alveoli, fewer ridge-like structures and thicker alveolar septa compared with control rats; these trends continued to day 14 (Fig. 1A).

Autophagosomes were observed in pulmonary cells of both control and BPD neonatal rats by TEM. Compared with the control group, the number of autophagosomes in the BPD group gradually increased, peaking at day 7 , at which time point autophagosome aggregates were visible (Fig. 1B).

Changes in STX17 expression in lung tissues of rats with BPD. Immunofluorescence staining and western blot analysis of STX17 protein expression demonstrated that it was expressed in nearly all pulmonary cells and was mainly cytoplasmic. On postnatal days 3 and 7, STX17 expression was significantly lower in the lungs of the BPD rats compared with the control rats, but afterwards the expression increased and reached a peak at day 14 (Fig. 2A, B and D). Consistent with the protein analysis, it was found that STX17 mRNA expression was significantly lower in the lung tissues of BPD rats compared with control rats on postnatal day 3. However, unlike STX17 protein expression, the mRNA expression of STX17 remained low (Fig. 2C).

Hyperoxia-induced changes in STX17 and autophagy protein expression in primary AT-II cells in vitro. To examine the effects of hyperoxia on AT-II cells directly, these cells were isolated from the lungs of control or BPD rats on postnatal day 1. Using light microscopy, the purified cells were elliptical in shape and had formed islets with paving stone-like changes (Fig. 3B). Using TEM, lamellar body structures were visible in the cells. These findings were consistent with the
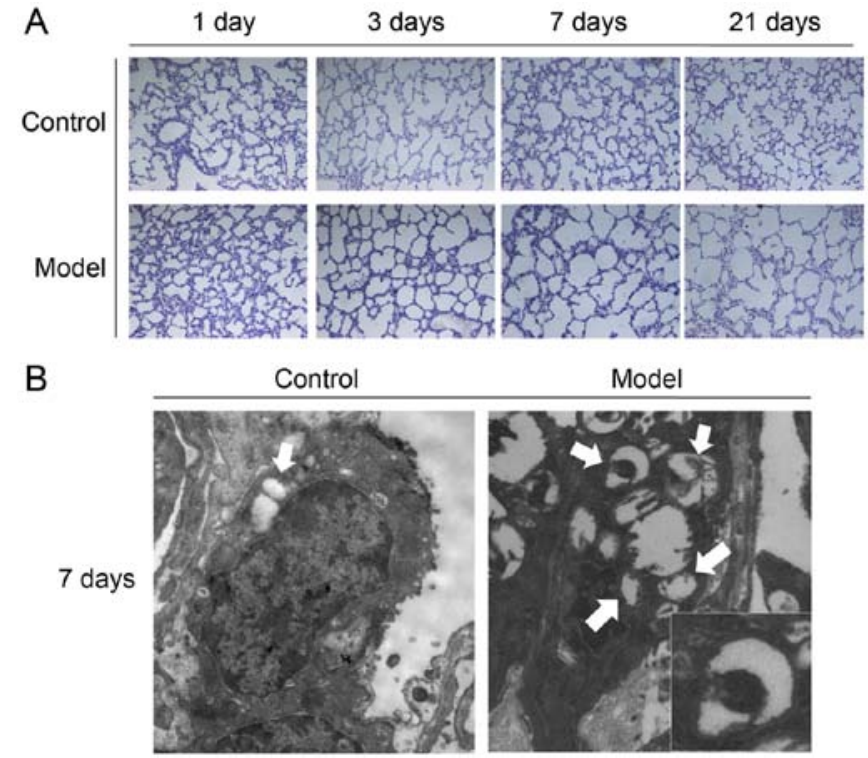

Figure 1. Structure of lung tissues from rats with hyperoxia-induced BPD. (A) Hematoxylin and eosin-stained lung sections from rats in the control and model groups on postnatal days 1, 3, 7 and 14. Magnification, x200. (B) Transmission electron microscopy of alveolar type II cells from rats in the control and model groups on postnatal day 7. White arrow indicates autophagosome aggregation. Magnification, x20,000 (local, x2).

morphological characteristics of AT-II cells (Fig. 3C). The identity of the cells was assessed by immunofluorescence staining for pro-surfactant, an AT-II cell-specific marker, which indicated that $\geq 90 \%$ of the cells (calculate by Image $\mathrm{J}$ ) exhibited positive cytoplasmic staining (Fig. 3A).

Western blot analysis was performed to examine STX17 protein expression. Lower STX17 protein expression was found in AT-II cells isolated from BPD rats compared with control rats on postnatal day 3 . In contrast, the expression levels of autophagy proteins LC3B-II, p62 and Lamp1, and the apoptotic protein cleaved caspase3, were all significantly higher in AT-II cells from BPD rats compared with control rats on postnatal day 7 , indicating that the BPD-induced change in STX17 protein expression preceded the effects on autophagyand apoptosis-related proteins (Fig. 4). Furthermore, while the same reduction was identified for STX17 mRNA expression in AT-II cells from BPD rats compared with control rats on postnatal days 3 and 7, there were no significant BPD-associated differences in the mRNA expression levels of LC3B, p62 or Lamp1 (Fig. 5). 
A

$$
\frac{1 \text { day }}{C \quad M} \quad \frac{3 \text { days }}{C \quad M} \quad \frac{7 \text { days }}{C \quad M} \frac{14 \text { days }}{C \quad M} \frac{21 \text { days }}{C \quad M}
$$

STX17

$\beta$-actin

B

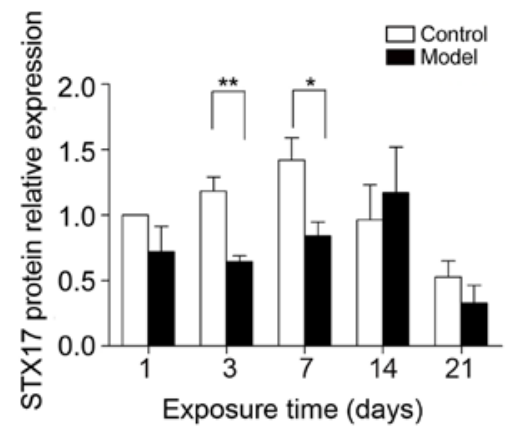

C

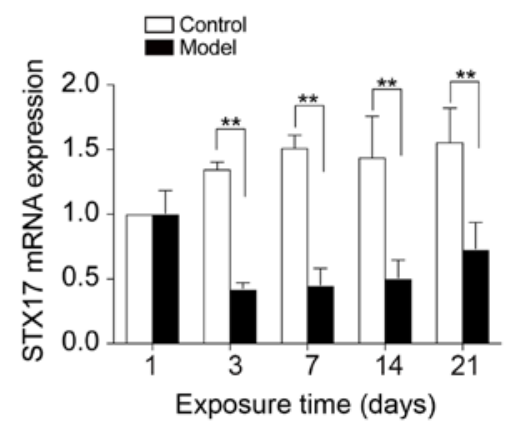

D
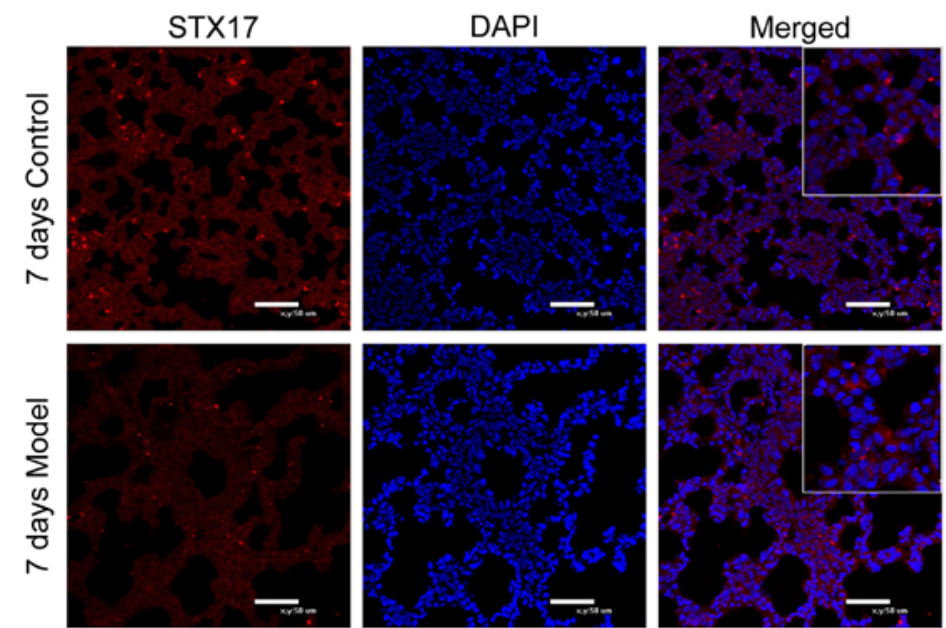

Figure 2. STX17 protein and mRNA expression levels in the lungs of BPD rats. (A) Western blot analysis of STX17 expression in the lung tissues of rats in the control group and BPD model group on the indicated days after birth and (B) semi-quantification of the results. (C) Reverse transcription-quantitative PCR analysis of STX17 mRNA expression in the lung tissues of rats in control and model group. (D) Immunofluorescence staining of STX17 protein in the lung tissues of rats in the control and model group (local, x2). Scale bar, $50 \mu \mathrm{m} .{ }^{*} \mathrm{P}<0.05,{ }^{* *} \mathrm{P}<0.01$. C, control; M, model; BPD, bronchopulmonary dysplasia; STX17, syntaxin 17.

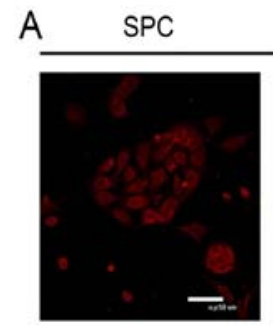

B

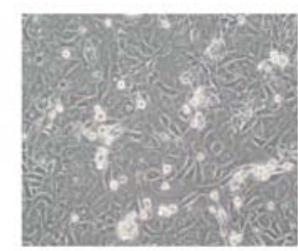

DAPI

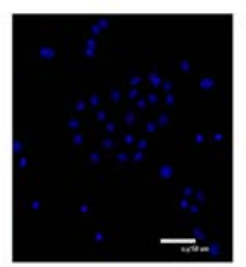

$\mathrm{C}$

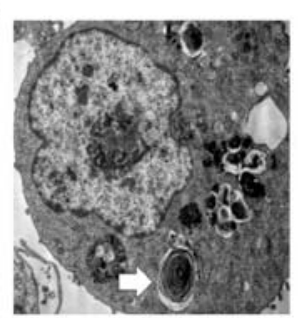

Figure 3. Characterization of primary AT-II cells. (A) Immunofluorescence staining of pro-SPC, an AT-II cell-specific marker, indicating that $\geq 90 \%$ of the cells were positive (red; magnification, x200). (B) Light microscopy of AT-II cells showing paving stone-like changes (magnification, $\mathrm{x} 200$ ). (C) Transmission electron microscopy images showing lamellar bodies in the cells (white arrow) Pro-SPC, surfactant; AT-II, alveolar type II (magnification, x20,000).

Next, the present study examined the effects of in vitro exposure to hyperoxia on primary AT-II cells isolated from BPD rats. The results indicated an early decrease in STX17 expression $(6 \mathrm{~h})$, followed by an increase in autophagy-related protein expression, in hyperoxic cells $(12 \mathrm{~h})$ compared with normoxic cells. In addition, STX17 expression was decreased by hyperoxia, reaching the lowest point at $6 \mathrm{~h}$, while LC3B-II and p62 protein expression levels were increased by hyperoxia, peaked after $12 \mathrm{~h}$ exposure and then gradually decreased (Fig. 6A).

Autophagy inhibitors reverse the effects of hyperoxia on primary AT-II cells in vitro. Whether modulation of autophagy affected AT-II cell survival under hyperoxia was also determined using AT-II cells exposed to hyperoxia in the presence or absence of the autophagy promoters RAPA $(5 \mu \mathrm{M})$ and $\mathrm{LiCl}$ $(5 \mathrm{mM})$ or the autophagy inhibitors 3-MA $(5 \mu \mathrm{M})$ and CQ $(5 \mu \mathrm{M})$. Exposure to RAPA promoted cell survival in hyperoxia condition (Fig. 6B) and decreased the expression levels of LC3B-II and cleaved caspase3 (Fig. 6C) compared with control cells. In addition, while STX17 protein expression was 


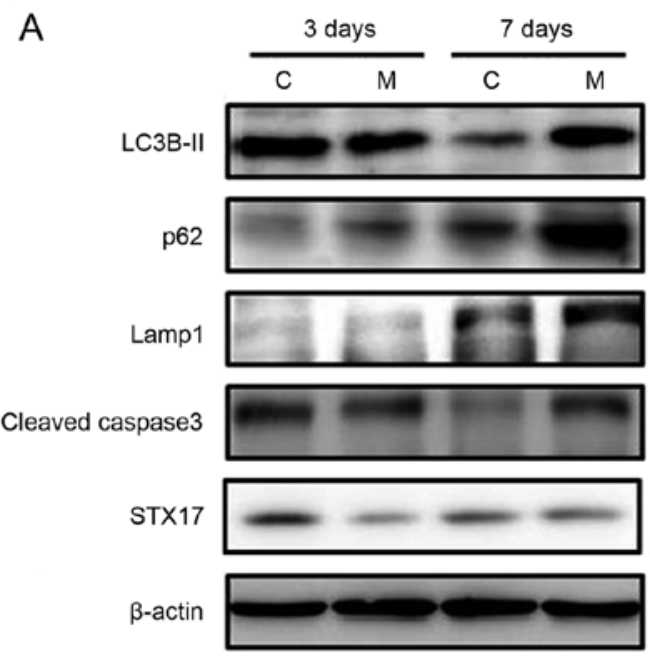

D

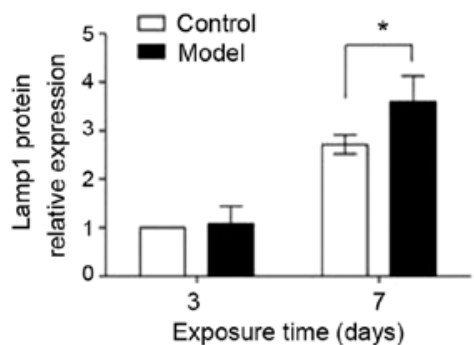

$\mathrm{E}$

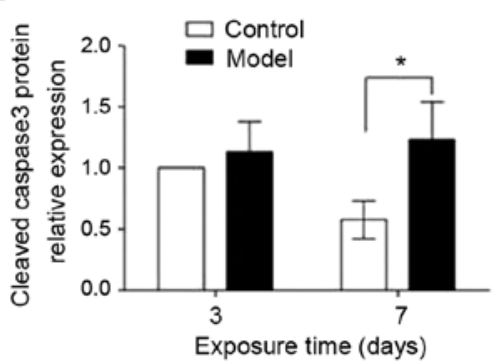

B

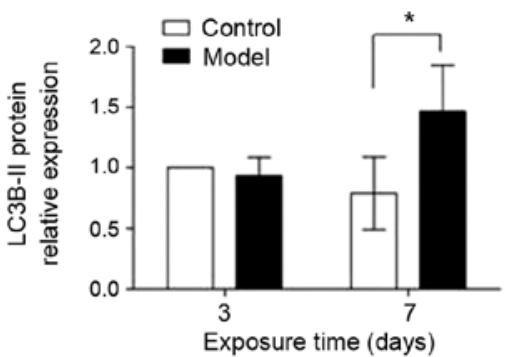

C

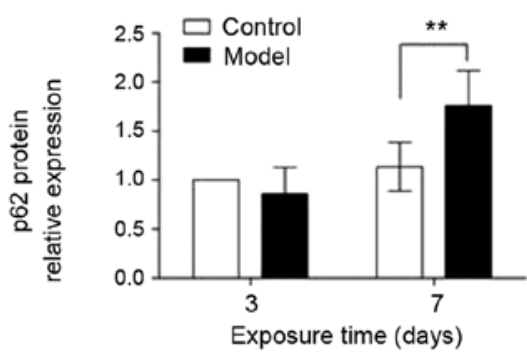

$\mathrm{F}$

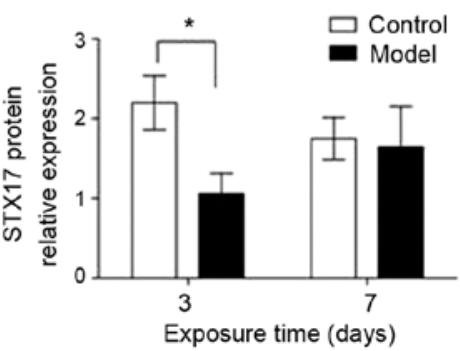

Figure 4. Expression of STX17 and autophagy- and apoptosis-related proteins in hyperoxic AT-II cells. (A) Western blot analysis of the (B) autophagy-related proteins LC3B-II, (C) p62 and (D) Lamp1, the apoptosis-related protein (E) cleaved caspase3 and (F) STX17 in primary AT-II cells. "P<0.05, ${ }^{* * *} \mathrm{P}<0.01$. AT-II, alveolar type II; STX17, syntaxin 17; LC3B, Microtubule-associated protein 1A/1B-light chain 3B; Lamp1, Lysosomal-associated membrane protein 1; C, control; M, model.
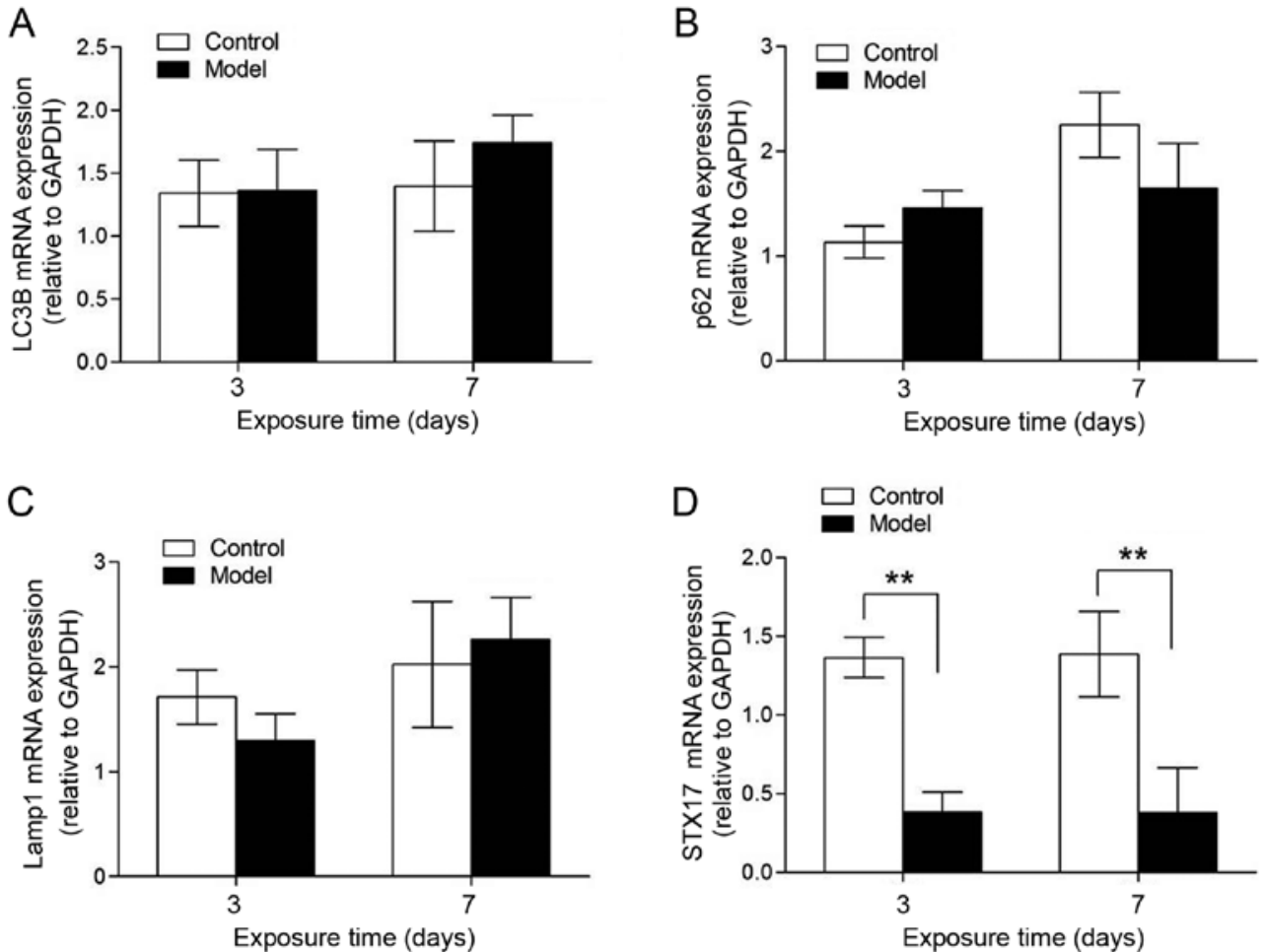

Figure 5. Expression of STX17 and autophagy- and apoptosis-related mRNAs in hyperoxia AT-II cells. RT-qPCR analysis of (A) LC3B, (B) p62, (C) Lamp1 and (D) STX17 mRNA expression levels in primary AT-II cells. ${ }^{* *} \mathrm{P}<0.01$. AT-II, alveolar type II; STX17, syntaxin 17; LC3B, Microtubule-associated protein 1A/1B-light chain 3B; Lamp1, Lysosomal-associated membrane protein 1. 
A

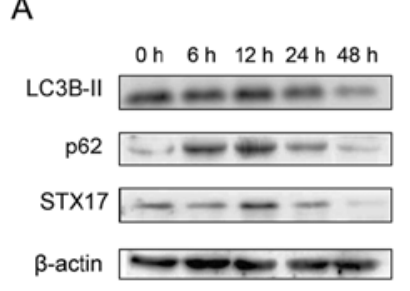

B
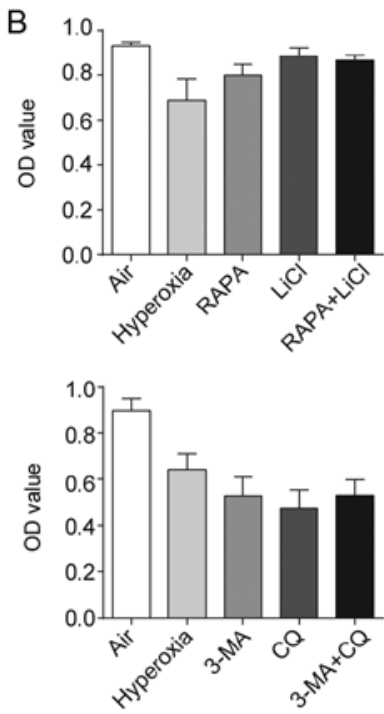
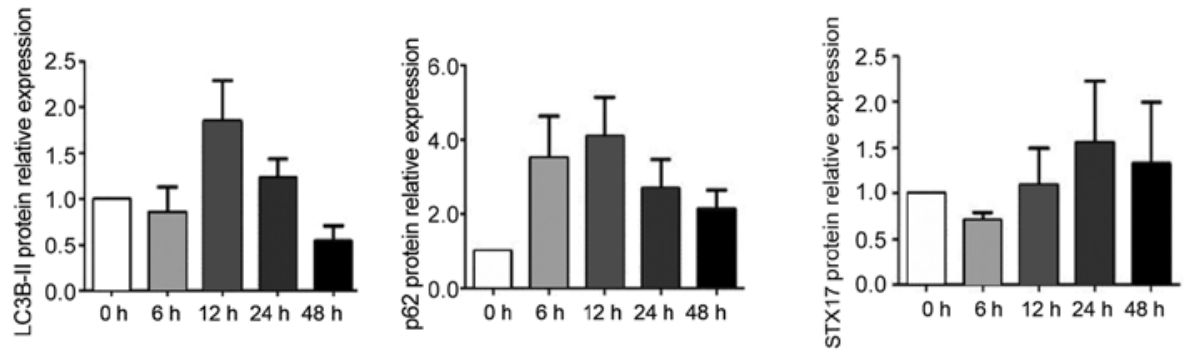

C
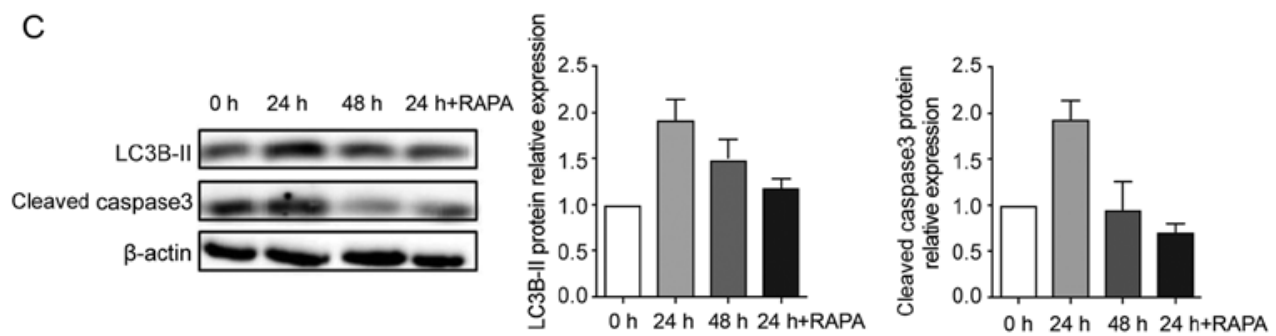

D

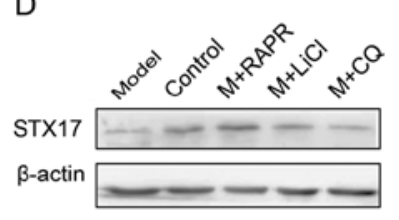

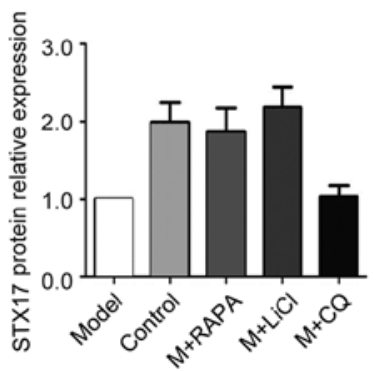

Figure 6. Expression of STX17 and autophagy- and apoptosis-related proteins in primary AT-II cells exposed to hyperoxia. (A) Western blot analysis of LC3B-II, p62 and STX17 in AT-II cells exposed to hyperoxia for the indicated times. (B) MTT proliferation assay of primary AT-II cells incubated with RAPA, LiCl, 3-MA and/or CQ. (C) Western blot analysis of LC3B-II and cleaved caspase3 in AT-II cells. (D) Western blot analysis of STX17 expression in AT-II cells incubated in the presence or absence of RAPA, LiCl or CQ. RAPA, rapamycin; 3-MA, 3-methyladenine; CQ, chloroquine; M, model; AT-II, alveolar type II; STX17, syntaxin 17; LC3B, Microtubule-associated protein 1A/1B-light chain 3B; Lamp1, Lysosomal-associated membrane protein 1; OD, optical density.

significantly decreased in cells after $6 \mathrm{~h}$ of hyperoxia, this was reversed by incubation with RAPA or $\mathrm{LiCl}$, but not with $\mathrm{CQ}$ (Fig. 6D). Thus, autophagy inhibitors restored the autophagic flux and promoted cell survival in AT-II cells exposed to hyperoxia.

\section{Discussion}

The development of BPD is influenced by multiple factors, including premature birth, oxidative stress, inflammation, stem cell damage, abnormal cell differentiation and trans-differentiation (2). However, the underlying molecular pathogenesis of BPD is not fully understood, and there are currently no safe and effective preventive or therapeutic clinical interventions (15). Thus, the development and analysis of animal models of BPD are important for investigating the pathogenesis of BPD.

Prolonged exposure of neonatal rats to high oxygen concentrations can influence alveolar and pulmonary vascular development and trigger pathological changes similar to those observed in human BPD $(16,17)$. The present study established a hyperoxia exposure model in neonatal rats that simulated the characteristic changes in BPD alveoli, and this was used to investigate the underlying mechanism of BPD (9).

Our previous studies reported that BPD in neonatal rats caused a block in the autophagic flux, causing increased pulmonary apoptosis and abnormal alveolar development $(9,18)$. Autophagy is crucial to embryonic development (19), and blockade of the pathway can result in aggregation of toxic abnormal proteins, which triggers an inflammatory response and eventually leads to cell apoptosis $(20,21)$. Abnormalities in the autophagic flux are speculated to be involved in the development of several pathologies, including cancer, neurodegenerative diseases, cardiovascular diseases and autoimmunity, as well as in aging (22). In respiratory system diseases, inhibition of the autophagic flux has been suggested to be associated with chronic inflammation and possibly the development of chronic obstructive pulmonary disease (23). Aberrant autophagy may also interfere with the epithelial-mesenchymal transition (EMT). When autophagic flux is blocked, epithelial cells can cause chronic inflammation due to clearance failure of a large amount of misfolded proteins, which is also one of the causes of EMT in epithelial cells (24), and reduced autophagic flux is known to be directly related to the development of pulmonary fibrosis (25). In our previous studies, it was shown that BPD caused a block in autophagic flux in pulmonary tissues, which was characterized by aggregation of autophagosomes and lysosomes, along with increased expression of the autophagosome marker LC3B-II, the autophagy substrate p62 and the lysosome marker Lamp1 $(9,18)$. These results are consistent with the present findings, as well as those reported by Zhang et al (26) and Sureshbabu et al (27), in which pulmonary epithelial cells exhibited autophagosome aggregation and increased LC3B-II expression after exposure to hyperoxia. It has also been shown that treatment with an 
autophagy inducer rescues the autophagic flux in pulmonary tissues under hyperoxia and improves lung development (9). However, the specific mechanism via which autophagic flux is blocked in BPD remains unknown.

Autophagy occurs via a series of steps, including the formation of autophagosomes, encapsulation of cellular cargo, binding and fusion of autophagosomes and lysosomes and the degradation of the lysosomal contents (11). Abnormalities occurring at any stage can influence the pathway function. Previous studies have reported that STX17 binds with two other SNARE proteins, Synaptosomal-associated protein 29 (SNAP29) and VAMP8, to enable the recognition and fusion of autophagosomes and lysosomes $(28,29)$. Thus, when STX17 expression or function is reduced, autophagosome-lysosome fusion is disrupted, resulting in aggregation of lysosomes and autophagosomes and inhibition of the autophagic flux (12). Furthermore, the SNAP29-STX17-VAMP8 complex is a key target for dysregulation of the autophagic flux occurring in numerous diseases. O-linked $\beta$-N-acetylglucosamine glycosylation of SNAP29 has been revealed to block autophagy and aggravate myocardial damage in type I diabetes by interfering with the SNAP29-STX17-VAMP8 complex (30). Another study reported that the toxicity of Coxsackie virus B3 may be related to reduced STX17 expression and blockade of the autophagic flux in HeLa cells (31). This study also revealed that overexpression of STX17 in HeLa cells restored autophagy and reduced apoptosis and the toxicity of Coxsackie virus (31). Certain bacteria, such as Legionella, can also block autophagy and increase apoptosis via the degradation of STX17 (32). In the present study, it was demonstrated that STX17 mRNA and protein expression levels were decreased at an early time point (postnatal day 3 ) in both the lung tissues and AT-II cells of neonatal rats with hyperoxia-induced BPD.

As alveolar stem cells, AT-II cells play an important role in alveolarization and recovery from alveolar damage, and AT-II cell dysfunction or defects may eventually result in alveolar dysplasia (33). In the present study, using an established model of hyperoxia-exposed AT-II cells, it was found that hyperoxia caused a decrease in STX17 expression followed by a significant increase in LC3B-II, p62 and cleaved caspase 3 expression, which is consistent with the results of the experiments with BPD pulmonary tissues. These findings suggested that the decrease in STX17 may be related to the subsequent blockade of autophagic flux following hyperoxia exposure. Furthermore, treatment of hyperoxia-exposed AT-II cells with the autophagy inducers RAPA and $\mathrm{LiCl}$ reduced the expression of LC3B-II and p62, partially restored the autophagic flux and may reduce apoptosis. In addition, the autophagy inducers restored STX17 expression. Therefore, it was speculated that hyperoxia may interfere with the autophagic flux of AT-II cells via the reduction in STX17 expression. Thus, restoring STX17 expression with pharmacological agents may be a potential therapeutic approach to alleviate BPD-induced defects in autophagy in AT-II cells.

In conclusion, the present results indicated that expression of STX17 was decreased in both pulmonary tissues and AT-II cells in response to hyperoxia during the crucial early neonatal stage of lung development. Moreover, the reduction in STX17 may be related to a subsequent block in autophagy. RAPA and
$\mathrm{LiCl}$ rescued the reduced STX17 expression and improved the autophagic flux; however, it is not fully understood whether the effect on autophagy was mediated via the change in STX17 expression itself or via another route. Therefore, further investigation is required to clarify the precise molecular mechanisms involved and to fully understand the pathogenesis of BPD.

\section{Acknowledgements}

The authors would like to thank the TEM technical support provided by Professor Fuhui Zhang from Department of Cytobiology, China Medical University, and the technical support in western blotting and RT-qPCR provided by Professor Zhihong Zong from Department of Biochemistry, China Medical University. The authors would also like to thank Dr Anne M. O'Rourke, from Liwen Bianji, Edanz Group China, for editing the English text of a draft of this manuscript.

\section{Funding}

This study was funded by the Natural Science Foundation of China (grant nos. 81901520 and 81571479), Basic research projects of Key Laboratory of Liaoning Provincial Department of Education (grant no. LZ2015070) and China Postdoctoral Science Foundation (grant no. 2019M661164).

\section{Availability of data and materials}

The datasets used and/or analyzed during the current study are available from the corresponding author on reasonable request.

\section{Authors' contributions}

JF and XX participated in the design of the study. XZ conceived of the study, and participated in its design. DiZ participated in its design and coordination and helped to draft the manuscript. SG participated in the immunoassays and performed the statistical analysis. DaZ participated in RT-qPCR and western blotting, and draft the manuscript. All authors read and approved the final manuscript.

\section{Ethics approval and consent to participate}

This study has passed and been approved by the Ethical Review of Scientific Research Projects from Shengjing Hospital of China Medical University (approval no. 2019PS321K).

\section{Patient consent for publication}

Not applicable.

\section{Competing interests}

The authors declare that they have no competing interests.

\section{References}

1. Thébaud B, Goss KN, Laughon M, Whitsett JA, Abman SH, Steinhorn RH, Aschner JL, Davis PG, McGrath-Morrow SA, Soll RF and Jobe AH: Bronchopulmonary dysplasia. Nat Rev Dis Primers 5: 78, 2019. 
2. Stoll BJ, Hansen NI, Bell EF, Shankaran S, Laptook AR, Walsh MC, Hale EC, Newman NS, Schibler K, Carlo WA, et al: Neonatal outcomes of extremely preterm infants from the NICHD Neonatal research network. Pediatrics 126: 443-456, 2010.

3. Jobe AH: The new bronchopulmonary dysplasia. Curr Opin Pediatr 23: 167-172, 2011.

4. Kinsella JP, Greenough A and Abman SH: Bronchopulmonary dysplasia. Lancet 367: 1421-1431, 2006.

5. Rock JR and Hogan BL: Epithelial progenitor cells in lung development, maintenance, repair, and disease. Annu Rev Cell Dev Biol 27: 493-512, 2011.

6. Rawlins EL: The building blocks of mammalian lung development. Dev Dyn 240: 463-476, 2011.

7. Willis KA, Siefker DT, Aziz MM, White CT, Mussarat N, Gomes CK, Bajwa A, Pierre JF, Cormier SA and Talati AJ Perinatal maternal antibiotic exposure augments lung injury in offspring in experimental bronchopulmonary dysplasia. Am J Physiol Lung Cell Mol Physiol 318: L407-L418, 2020.

8. Choi AM, Ryter SW and Levine B: Autophagy in human health and disease. N Engl J Med 368: 651-662, 2013.

9. Zhang D, Wu L, Du Y, Zhu Y, Pan B, Xue X and Fu J: Autophagy inducers restore impaired autophagy, reduce apoptosis, and attenuate blunted alveolarization in hyperoxia-exposed newborn rats. Pediatr Pulmonol 53: 1053-1066, 2018.

10. Yim WW and Mizushima N: Lysosome biology in autophagy. Cell Discov 6: 6, 2020.

11. Itakura E and Mizushima N: Syntaxin 17: The autophagosomal SNARE. Autophagy 9: 917-919, 2013.

12. Hubert V, Peschel A, Langer B, Gröger M, Rees A and Kain R Lamp-2 is required for incorporating syntaxin-17 into autophagosomes and for their fusion with lysosomes. Biol Open 5 : 1516-1529, 2016

13. Zhu Y,Fu J, Yang H, Pan Y, Yao L and Xue X: Hyperoxia-induced methylation decreases RUNX3 in a newborn rat model of bronchopulmonary dysplasia. Respir Res 16: 75, 2015.

14. Livak KJ and Schmittgen TD: Analysis of relative gene expression data using real-time quantitative PCR and the 2(-Delta Delta C(T)) method. Methods 25: 402-408, 2001

15. Jain D and Bancalari E: Bronchopulmonary dysplasia: Clinical perspective. Birth Defects Res A Clin Mol Teratol 100: 134-144, 2014.

16. Manji JS, O'Kelly CJ, Leung WI and Olson DM: Timing of hyperoxic exposure during alveolarization influences damage mediated by leukotrienes. Am J Physiol Lung Cell Mol Physiol 281: L799-L806, 2001.

17. Chen CM, Wang LF, Chou HC, Lang YD and Lai YP Up-regulation of connective tissue growth factor in hyperoxia-induced lung fibrosis. Pediatr Res 62: 128-133, 2007.

18. Zhao X, Shi Y, Zhang D, Tong X, Sun Y, Xue X and Fu J: Autophagy inducer activates Nrf2-ARE pathway to attenuate aberrant alveolarization in neonatal rats with bronchopulmonary dysplasia. Life Sci 252: 117662, 2020.

19. Tsukamoto S, Kuma A, Murakami M, Kishi C, Yamamoto A and Mizushima N: Autophagy is essential for preimplantation development of mouse embryos. Science 321: 117-120, 2008.
20. Zhang Q, Kang R, Zeh HJ III, Lotze MT and Tang D: DAMPs and autophagy: Cellular adaptation to injury and unscheduled cell death. Autophagy 9: 451-458, 2013.

21. Boya P, Gonzalez-Polo RA, Casares N, Perfettini JL, Dessen P, Larochette N, Métivier D, Meley D, Souquere S, Yoshimori T, et al: Inhibition of macroautophagy triggers apoptosis. Mol Cell Biol 25: 1025-1040, 2005.

22. Liu J and Debnath J: The evolving, multifaceted roles of autophagy in cancer. Adv Cancer Res 130: 1-53, 2016.

23. Vij N, Chandramani-Shivalingappa P, Van Westphal C, Hole R and Bodas M: Cigarette smoke-induced autophagy impairment accelerates lung aging, COPD-emphysema exacerbations and pathogenesis. Am J Physiol Cell Physiol 314: C73-C87, 2018.

24. Lu WH, Wang G, Li Y, Li S, Song XY, Wang XY, Chuai M, Lee KK, Cao L and Yang X: Autophagy functions on EMT in gastrulation of avian embryo. Cell Cycle 13: 2752-2764, 2014

25. Meng Y, Pan M, Zheng B, Chen Y, Li W, Yang Q, Zheng Z, Sun N, Zhang Y and Li X: Autophagy attenuates angiotensin ii-induced pulmonary fibrosis by inhibiting redox imbalance-mediated NOD-like receptor family pyrin domain containing 3 inflammasome activation. Antioxid Redox Signal 30: 520-541, 2018.

26. Zhang L, Zhao S, Yuan LJ, Wu HM, Jiang H, Zhao SM, Luo G and Xue XD: Autophagy regulates hyperoxia-induced intracellular accumulation of surfactant protein $\mathrm{C}$ in alveolar type II cells. Mol Cell Biochem 408: 181-189, 2015

27. Sureshbabu A, Syed M, Das P, Janér C, Pryhuber G, Rahman A, Andersson S, Homer RJ and Bhandari V: Inhibition of regulatory-associated protein of mechanistic target of rapamycin prevents hyperoxia-induced lung injury by enhancing autophagy and reducing apoptosis in neonatal mice. Am J Respir Cell Mol Biol 55: 722-735, 2016.

28. Viret $\mathrm{C}$ and Faure M: Regulation of syntaxin 17 during autophagosome maturation. Trends Cell Biol 29: 1-3, 2019.

29. Shen Q, Shi Y, Liu J, Su H, Huang J, Zhang Y, Peng C, Zhou T, Sun Q, Wan W and Liu W: Acetylation of STX17 (syntaxin 17) controls autophagosome maturation. Autophagy: 1-13, 2020 (Epub ahead of print)

30. Supplemental therapeutic oxygen for prethreshold retinopathy of prematurity (Stop-Rop), a randomized, controlled trial. I: Primary outcomes. Pediatrics 105: 295-310, 2000.

31. Tian L, Yang Y, Li C, Chen J, Li Z, Li X, Li S, Wu F, Hu Z and Yang Z: The cytotoxicity of coxsackievirus B3 is associated with a blockage of autophagic flux mediated by reduced syntaxin 17 expression. Cell Death Dis 9: 242, 2018.

32. Arasaki $\mathrm{K}$ and Tagaya M: Legionella blocks autophagy by cleaving STX17 (syntaxin 17). Autophagy 13: 2008-2009, 2017.

33. Del Riccio V, van Tuyl M and Post M: Apoptosis in lung development and neonatal lung injury. Pediatr Res 55: 183-189, 2004.

This work is licensed under a Creative Commons Attribution-NonCommercial-NoDerivatives 4.0 International (CC BY-NC-ND 4.0) License. 